\title{
Il catetere venoso centrale nel paziente con scompenso cardiaco in emodialisi: una analisi retrospettiva
}

\author{
B. Ricciardi ${ }^{1}$, A. Pontoriero ${ }^{1}$, A. Ragusa ${ }^{1}$, C.A. Riccardi ${ }^{1}$, A. Granata ${ }^{2}$ \\ ${ }^{1}$ U.O.C. Nefrologia e Dialisi P.O. "Fogliani”, Milazzo \\ ${ }^{2}$ U.O.C. Nefrologia e Dialisi P.O. "San Giovanni di Dio”, Agrigento
}

CONTROL VENOUS CATHETER AMONG PATIENTS WITH HEART FAILURE IN HAEMODIALYSIS: A RETROSPECTIVE ANALYSIS

ABstract. Vascular access for hemodialysis in patient with heart failure is a complex clinical issue, that requires combined technical skills from nephrological and cardiological field for its full understanding.

In these patients, in fact a native proximal vascular access can elicite a long series of effects on cardiac function, potentially degrading performance and patient outcomes. In such patients is therefore possible that the placement of a central venous catheter represents a solution less problematic.

This article presents a monocentric experience and some clinical examples, useful to investigate this peculiar condition.

Key Words. Hemodialysis, Artero-venous fistula, Vascular access, Heart failure

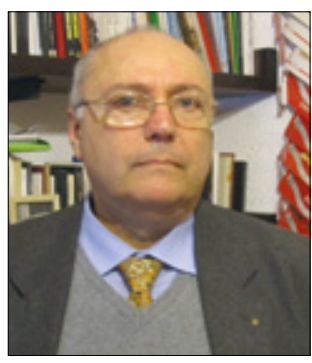

Biagio Ricciardi

\section{Introduzione}

Lo scompenso cardiaco è una sindrome clinica complessa, originante da una affezione cardiaca strutturale o funzionale, caratterizzata da sintomi specifici quali dispnea da sforzo e/o a riposo, ortopnea, astenia, ritenzione idrica, distensione delle vene giugulari

(Fig. 1), epatomegalia, ascite, edemi declivi, versamento pleurico ed edema polmonare.

Il rene ha un ruolo determinante nelle prime fasi dell'adattamento allo scompenso cardiocircolatorio, controbilanciando l'iperattivazione dei sistemi sodioritentivi e vasocostrittori (sistema simpatico, sistema renina-angiotensina-aldosterone, vasopressina) e di quelli vasodilatatori e natriuretici (dopamina, prostaglandine, peptidi natriuretici). Proprio la prevalenza dei primi sui secondi causa la riduzione del flusso plasmatico renale ed il conseguente aumento della frazione di filtrazione, all'origine della disfunzione renale. In caso di pre-esistente nefropatia, tali alterazioni sono più precoci e rilevanti (1-3). Le malattie cardiovascolari sono la causa di mortalità più frequente tra i pazienti dializzati, contando per quasi il 50\% dei decessi. L'ischemia miocardica e lo scompenso cardiaco determinano tra questi il $20 \%$ dei ricoveri, con una prevalenza di scompenso cardiaco da 10 a 30 volte maggiore rispetto alla popolazione generale (4). $\mathrm{La}$ necessità di un ricovero ospedaliero per scompenso cardiaco riduce la sopravvivenza al $12.5 \%$, nettamente inferiore rispetto a quanto osservato in caso di semplice sovraccarico idrico (20.2\%) o di un solo episodio di edema polmonare (associato ad una sopravvivenza del $21.3 \%$ )

In questo panorama sicuramente drammatico, il nefrologo è chiamato ad avviare un numero sempre maggiore di pazienti a rischio di scompenso (anziani, diabetici, cardiopatici) al trattamento extracorporeo. Questi pazienti rendono particolarmente complessa la scelta dell'accesso vascolare da confezionare: fistola arterovenosa, graft protesico o catetere venoso centrale "long term"?

Il patrimonio vascolare del paziente a rischio di scompenso è spesso altamente compromesso, obbligando a scelte chirurgiche aggressive che prediligono il confezionamento di accessi prossimali. La gestione clinica dell'accesso è ulteriormente complicata dallo stato pressorio di tali pazienti e dalla necessità di ricorrere a 


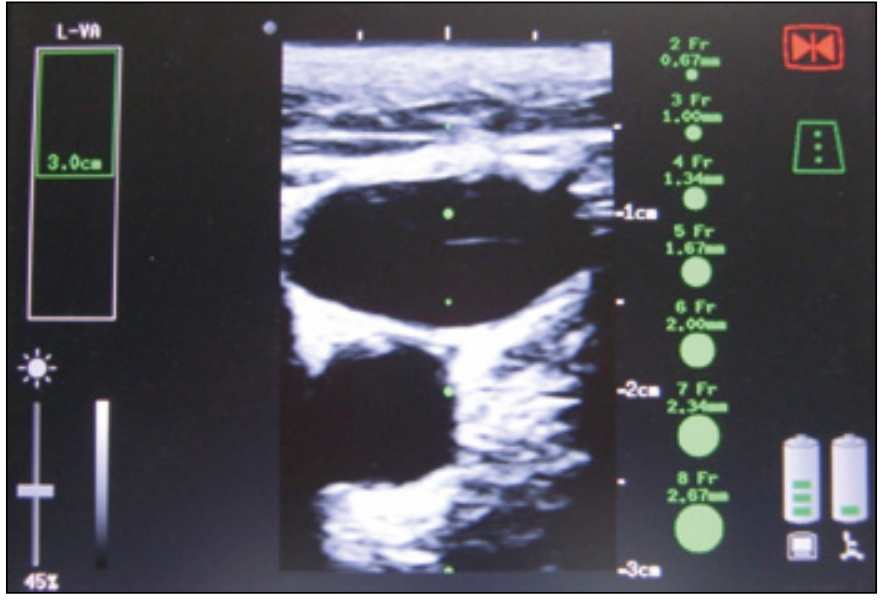

Fig. 1 - Paziente ultra-sessantacinquenne, vena giugulare di grandi dimensioni, anteroposta alla carotide.

dosaggi elevati di diuretici, così da mettere a rischio la buona riuscita del confezionamento di una FAV distale a causa di una scarsa vis a tergo e del conseguente rischio di trombosi, inadeguato sviluppo, flussi ematici insufficienti.

In tal caso la FAV prossimale, caratterizzata da migliori percentuali di successo e ad oggi ritenuta la soluzione preferenziale per l'accesso vascolare in tali pazienti, comporterà un rischio elevato di peggioramento dello scompenso.

Iwashima Y, et al (5) hanno osservato tali effetti, misurandoli sia mediante studi ecocardiografici seriati che mediante i livelli circolanti di ANP (Atrial Natriuretic Peptide) e pro-BNP (Brain Natriuretic Peptide) entrambi marcatori di disfunzione cardiaca. Secondo tali Autori la semplice creazione dell'accesso artero-venoso incrementa la gittata cardiaca del 15\%, il diametro telediastolico del ventricolo sinistro del $4 \%$, la disfunzione diastolica del ventricolo sinistro, incrementando altresì sia la secrezione di ANP (direttamente correlata col volume di carico) che la secrezione di pro-BNP (verosimilmente correlata alla disfunzione diastolica del ventricolo sinistro).

In un altro recente lavoro, Yigla et al (6) hanno dimostrato inoltre che nellimmediato post-FAV si osserva un incremento dei valori pressori polmonari, e che tale adattamento, unito all'aumento della gittata cardiaca, riduce la sopravvivenza, seppure mostrando la tendenza a regredire dopo la chiusura della FAV.

In una casistica di 96 pazienti uremici, portatori di FAV, tra quelli portatori di accesso prossimale (7), vengono osservati numerosi casi di scompenso cardiaco ad alta gittata (9.6\%).

Se è frequente osservare come le modifiche emodinamiche causate dalla fistola possano passare clinicamente inosservate, è dato comune che portate superiori ai $2 \mathrm{~L} /$ min comportino un rischio moderato di scompenso cardiaco, mentre tale rischio diviene francamente elevato per valori superiori ai $4 \mathrm{~L} / \mathrm{min}$. Ovviamente al riguardo non sembrano esserci differenze significative tra una FAV nativa e un graft protesico.

Altro indicatore di rischio di scompenso cardiaco è il rapporto di flusso tra l'accesso $(\mathrm{Qa})$ e la gittata cardiaca (CO). Un rapporto $\mathrm{Qa} / \mathrm{CO}=0.3$ indica un "rischio significativo" di scompenso cardiaco ad alta gittata.

Se tale rapporto addirittura supera la soglia di 0.3 , si impone la riduzione chirurgica del flusso della FAV mediante tecniche di "banding", cioè di riduzione chirurgica del calibro del vaso efferente teso a ridurne il flusso, seppure con il rischio di incidente trombotico fatale per l'accesso.

In caso di inefficacia di tale intervento, risulterà allora indispensabile la chiusura, in questo caso intenzionale, della FAV, con ricorso obbligato all'impianto di un catetere venoso centrale "long term" ovvero, ove possibile, al trattamento dialitico peritoneale.

Quanto sin qui descritto acquista pieno valore in pazienti non affetti da scompenso cardiaco clinicamente evidente. Gli effetti avversi della FAV sull'apparato cardiovascolare sono ovviamente molto più rapidi e pesanti in pazienti già affetti da manifesto scompenso, per i quali sono da attendersi pesanti incrementi del precarico anche per $\mathrm{Qa} / \mathrm{CO}<0.3$.

Un monitoraggio prospettico per la rilevazione di un eventuale scompenso cardiaco da alta gittata, è raccomandato (8), soprattutto nei confronti di soggetti ad alto rischio (età avanzata, cardiopatici e portatori di FAV prossimale). Le linee guida K-DOQI suggeriscono un monitoraggio mediante esame clinico a cadenza settimanale, accompagnato da sorveglianza strumentale Doppler mensile in presenza di significativa dilatazione della FAV, pulsazione rigida e thrill sospetto per alto flusso vascolare.

\section{Perché quindi il catetere in prima istanza?}

Un catetere venoso centrale correttamente posizionato, e pertanto non di disturbo alla meccanica valvolare, è totalmente ininfluente sulla cinetica cardiaca. Questo fa dei CVC permanenti l'unica alternativa valida in caso di necessità di trattamento extracorporeo in pazienti affetti da scompenso in classe III-IV NYHA (9).

La possibilità di combinare la precisione della venipuntura eco-guidata, con la sicurezza del controllo radioscopico durante il percorso intratoracico in itinere, cosiddetta Tecnica Ecoscopica è in grado di ridurre al minimo i ri- 
schi di impianto, che risultano praticamente azzerati, di ridurre i tempi di intervento, ed incrementare il senso di sicurezza dell'operatore con una conseguente ottimale compliance del paziente.

Limpianto dei CVCcon tale tecnica evita pressoché totalmente, le sollecitazioni meccaniche intra-cardiache, l'impegno della valvola tricuspide, il rischio di imboccare false strade in caso di anomalie di percorso.

I recenti progressi tecnologici nel campo dei CVC ne hanno d'altra parte notevolmente migliorato il comfort, al punto che è esperienza diffusa quanto i pazienti anziani tendano a preferire tali dispositivi al confezionamento di una FAV.

Il rischio infettivo, associato nei pazienti emodializzati alla mortalità cardiovascolare e da stroke (10), è certamente un tallone d'Achille per tali accessi. Tale rischio necessita di un approccio multidimensionale, che passi per una ottimale gestione infermieristica in corso di connessione e (deconnessione non è usato nel nostro gergo) sconnessione dal monitor, per una corretta formazione culturale del paziente stesso e del personal e dalla necessità di trattare il CVC alla stessa stregua e con la stessa cura di un catetere peritoneale.

Nella nostra esperienza abbiamo trattato 135 pazienti in classe III-IV NYHA, in un periodo di 13 anni, dal 1999 ad oggi.

In tali pazienti sempre mediante Tecnica Ecoscopica sono stati impiantati:

- CVC temporanei Mahurkar (Covidien), convertiti a 30 giorni in "long term" fino al 2005.

- Long term tunnellizzati "Permcath" (Covidien) dal 2006 sino al 2010.

- Long term Palindrome (Covidien) dal 2010 sino ad oggi.

Questa coorte, composta soprattutto da pazienti "late referral”, da sottoporre a trattamento extracorporeo in urgenza, si è caratterizzata per una mortalità iniziale piuttosto alta pur non osservando correlazioni tra eventuali complicanze da CVC e mortalità secondo quanto indicato:

- il $70 \%$ a sei mesi dall'inizio del trattamento,

- $\quad$ il $20 \%$ nei successivi 6 mesi di trattamento,

- il $10 \%$, in un periodo variabile da 1 a tre anni di trattamento, si ripete senza comunque manifestare problematiche riconducibili al Catetere Venoso Centrale.

L'aspettativa di vita di questi pazienti è stata quindi tale da non superare in nessun caso i tre anni dall'inizio della terapia emodialitica. Ciò può ha concorso a giustificare il diffuso atteggiamento di ricorrere pressoché sistematicamente all'utilizzo di cateteri venosi in tale tipologia di pazienti.

Riportiamo di seguito riportato tre casi clinici, che a no- stro giudizio risultano esemplificativi dei possibili effetti positivi del trattamento extracorporeo mediante CVC sulla storia clinica di tali pazienti.

\section{Paziente 1}

Paziente grande obeso (peso corporeo $>120 \mathrm{~kg}$ ) di sesso maschile, 79 anni, affetto da diabete tipo 2 corretto mediante l'infusione di insulina ai pasti, dislipidemico, ipertensione in trattamento farmacologico, uremia terminale "late referral".

Un ecocardiogramma eseguito prima della terapia dialitica mostrava: "Ventricolo Sinistro severamente dilatato, ipocinetico, con severa depressione della funzione sistolica globale (frazione di eiezione 30\%). Lieve ipertrofia parietale concentrica. Dilatazione biatriale. Ventricolo Destro ai limiti. Cuspidi aortiche fibrocalcifiche con lieve aumento dei gradienti transvalvolari. Al Doppler insufficienza mitralica di grado moderato, tricuspidalica di grado lieve, Pressione polmonare non valutabile. Non versamento pericardico in atto.

Viene iniziato trattamento sostitutivo con Emodiafiltrazione, previo impianto di CVC "long term" tunnellizzato in vena giugulare destra, con progressiva riduzione del peso corporeo da 120 a $100 \mathrm{~kg}$ in 48 giorni e normalizzazione dei valori glicemici e della dislipidemia.

L'ecocardiogramma a 60 giorni evidenziava: "Ventricolo sinistro di dimensioni endocavitarie normali, con lieve ipertrofia delle pareti, eucinesia segmentaria con funzione globale di pompa conservata. Frazione di eiezione $55 \%$ circa (migliorata dell' $80 \%$ complessivamente). Atrio sinistro lievemente dilatato. Ventricolo destro eucinetico, di dimensioni nella norma. Atrio sinistro nei limiti. Setti integri. Foglietti pericardici iperecogeni, presenza di versamento pericardico di entità media.

L'ecocardiogramma a 24 mesi di distanza evidenziava una funzione di pompa del ventricolo sinistro globalmente conservata, con FE di circa $55 \%$.

\section{Paziente 2}

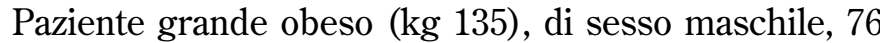
anni, con coronaropatia multivasale sottoposta a PTCA duplice + stent nel 2002, impianto di ICD bicamerale nel 2008 ed evoluzione in cardiomiopatia dilatativa postischemica, uremia terminale, dislipidemia.

L'Ecocardiogramma pre-dialisi evidenziava: "Radice aortica fibro-calcifica, lievemente ectasica $(41 \mathrm{~mm})$. Valvola aortica tricuspide, con normale separazione sistolica. Ventricolo sinistro dilatato (Diametro tele sistolico: $56 \mathrm{~mm}$; diam. tele diastolico: $208 \mathrm{~mL}$ ), con moderata ipertrofia settale e lieve ipertrofia a carico della parete antero-laterale, globalmente ipocinetico, con discinesia del setto distale, marcata ipocinesia della parete latera- 
le, discinesia della porzione basale inferiore, ipocinesia della porzione media inferiore, marcata ipocinesia della porzione medio-basale anteriore. Compromissione della funzione globale di pompa con FE 30\%. Lieve dilatazione bi-atriale. Apparati valvolari atrio-ventricolari fibrotici. Pericardio indenne.

Viene impiantato, in condizioni generali particolarmente scadenti, un CVC Palindrome 36/19 (Fr 14.5 mm 4.8) in giugulare destra ed inizia trattamento di HDF on line. L'ecocardiogramma dopo 30 giorni di terapia dialitica riporta: Ventricolo sinistro moderatamente dilatato (Diametro tele sistolico: $59 \mathrm{~mm}$; diam. tele diastolico: $170 \mathrm{~mm}$ - complessivamente ridotto del $30 \%$ ), eucinesia del SIV posteriore, della parete inferiore e posteriore (segmento medio-basale). Moderata ipertrofia del SIV (mm 17). Severa depressione della funzione sistolica globale (FE 35\% complessivamente migliorato del 20\%). Lieve dilatazione biatriale. Ventricolo destro ai limiti. Lieve dilatazione dell'aorta ascendente ( $\mathrm{mm} 43)$. Insufficienza aortica, mitralica e tricuspidalica lievi. PAPs circa $30 \mathrm{mmHg}$. Non versamento pericardico in atto.

Questi due casi, in cui comunque si è osservato un incremento della frazione di eiezione, si prestano a commentare quella che è oramai esperienza comune di tanti Centri Nefrologici, per la quale pazienti affetti da scompenso cardiaco refrattario ai diuretici trovano nel trattamento extra-corporeo una vera "macchina del tempo", capace di riportarne l'età biologica indietro negli anni. Quello che si avvera in tali pazienti è che l'efficace correzione della volemia risolve rapidamente $\mathrm{i}$ fenomeni di iponatremia, iperaldosteronismo, disventilazione ed ipertensione polmonare tipici dello scompenso congestizio, restituendo ad essi una condizione di omeostasi non altrimenti raggiungibile con la terapia farmacologica.

Tali pazienti presentano poi ulteriori temi di interesse per i nefrologi, i quali attuando una corretta terapia con ESA, correggendo l'equilibrio acido-base, le disionie e l'iperparatiroidismo, svolgono una preziosa opera "di contorno" allo scompenso, che ristabilisce un equilibrio di insieme e può far letteralmente rifiorire pazienti altrimenti facilmente ritenuti come oramai perduti.

\section{Paziente 3}

Paziente di sesso femminile, di 43 anni, sottoposta quattro anni addietro a trapianto di rene, ben funzionante, e portatrice di FAV distale avambraccio sinistro. La paziente, in terapia con bisoprololo $2.5 \mathrm{mg}$ prima del trapianto, ne ha incrementato il dosaggio a $5 \mathrm{mg}$ progressivamente per il riscontro di aritmie. Non sono presenti alterazioni significative agli ECG estemporanei.

Esegue ECG holter, che evidenzia ritmo sinusale a frequenza cardiaca media di $68 \mathrm{~b} / \mathrm{min}$ (minima di $46 \mathrm{~b}$ / min) e ben 10538 battiti ectopici ventricolari, di cui 9461 isolati, 536 coppie e 2 runs di tachicardia ventricolare non sostenuti (massimo di 4 battiti).

La paziente si ricovera per algie al braccio sede della FAV ed insorgenza di palpitazioni e precordalgie, non giudicate significative all'ECG.

Un nuovo ECG holter evidenzia ritmo sinusale a frequenza media di 67 b/m' (min di 52 e mass di 91 b/min, con normale conduzione atrioventricolare e intraventricolare. Sono però presenti ben 12252 battiti ectopici ventricolari (incrementati del $30 \%$ rispetto a due anni prima), comprendenti 118 coppie e 5 episodi di tachicardia ventricolare non sostenuta.

Esegue quindi un ecocardiogramma, che riporta un lieve incremento dello spessore del SIV, una conservata cinetica segmentaria, normali indici di funzione sistolica del VS (FE di circa 60\%), ridondanza dei lembi valvolari mitralici con prolasso sistolico di entrambi i lembi. Sono inoltre presenti un lieve ingrandimento atriale sinistro $\left(22 \mathrm{~cm}^{2}\right)$, insufficienza mitralica lieve moderata ed insufficienza tricuspidalica lieve.

Si opta quindi per la chiusura chirurgica della FAV distale sinistra (classica), al momento ben funzionante.

L'ECG Holter eseguito dopo una settimana riporta 3303 ectopie ventricolari, delle quali 3059 isolate, con una riduzione delle ectopie ventricolari di oltre il $70 \%$. Un ecocardiogramma eseguito dopo soli sei giorni dalla chiusura della FAV evidenzia la riduzione della dilatazione atriale sinistra $\left(21 \mathrm{~cm}^{2}\right)$.

\section{Conclusioni}

Il paziente affetto da scompenso cardiaco refrattario ai diuretici trova grande giovamento nell'ultrafiltrazione extracorporea. Il nefrologo viene educato a sviluppare veri e propri "sensi di colpa" nei confronti dei cateteri venosi centrali a permanenza, che vive come elemento di allarme e segnali di fallimento della propria politica degli accessi. La nostra esperienza ci spinge a valutare con senso critico tale atteggiamento, e questa piccola casistica vuole pertanto sollevare l'attenzione del lettore su un tema di comune riscontro: il paziente più strettamente "cardiologico" rappresenta un'eccezione alla regola secondo la quale l'accesso venoso mediante FAV debba necessariamente rappresentare la prima scelta! E nostra opinione infatti che lo scompenso cardiaco in stadio NYHA IV-V, refrattario ai diuretici tanto da richiedere ultrafiltrazione extracorporea, abbia indicazione all'accesso elettivo mediante CVC Long Term in prima istanza o, ove possibile, al ricorso precoce alla dialisi peritoneale. Una rivalutazione del paziente a distanza 
di 6-12 mesi, per una ri-classificazione NYHA, potrà poi permettere di affrontare con maggior serenità e contezza la decisione di lungo termine circa l'accesso vascolare in tali particolari pazienti.

Presso il nostro Centro Nefrologico pertanto tutti i pazienti in attesa di confezionamento di FAV vengono sottoposti ad ecocardiogramma ed Holter ECG, onde selezionare quei soggetti per i quali una FAV possa rappresentare un ulteriore insulto alla funzione cardiaca.

Gli stessi esami vengono quindi ripetuti in fase di followup a 30 giorni e a 12 mesi dall'intervento, onde valutare l'evoluzione del muscolo cardiaco ed eventualmente intervenire farmacologicamente, con un cambio di strategia dialitica e/o di accesso vascolare, chiudendo chirurgicamente una FAV ed impiantando un CVC long term, nei pazienti che mostrino rapida evolutività verso lo scompenso ad alta gittata.

\section{Riassunto}

L'accesso vascolare per emodialisi nel paziente affetto da scompenso cardiaco è un tema clinico complesso, che necessita di competenze tecniche nefrologiche e cardiologiche per una sua piena comprensione.

In tali pazienti infatti il confezionamento di un accesso vascolare nativo prossimale presenta una lunga serie di effetti sulla funzione cardiaca, potenzialmente in grado di peggiorare la performance e la prognosi del paziente. In tali pazienti è quindi possibile che il posizionamento di un catetere venoso centrale rappresenti una soluzione meno problematica.

Questo articolo presenta una casistica monocentrica ed alcuni casi clinici esemplificativi, utili ad indagare tale peculiare condizione.

Parole Chiave. Catetere venoso centrale, Fistola arterovenosa, Scompenso cardiaco

\section{Indirizzo degli Autori:}

Dr. Biagio Ricciardi, MD

U.O.C. Nefrologia e Dialisi P.O. "Fogliani”, Milazzo

C/da Baglio $n^{\circ} 33$ Villaggio Sant’Agata

98166 Messina

biagioricciardi@asp.messina.it

\section{Bibliografia}

1. Andreoli TE. Pathogenesis of renal sodium retention in congestive heart failure. Mineral Electrolyte Metab 1999; 25: $1-20$.

2. Cody RJ, Ljungman S, Covit AB, et al. Regulation of glomerular filtration rate in chronic congestive heart failure patients. Kidney Int 1988; 34: 361-7.

3. Schrier RW. Pathogenesis of sodium and water retention in hight-output cardiac failure, nephritic syndrome, cirrhosis, and pregnancy. N Engl J Med 1988; 319: 1127.

4. Banerjee D, Ma JZ, Collins AJ, Herzog CA. Long-term survival of incident hemodialysis patients who are hospitalized for congestive heart failure, pulmonary edema, or fluid overload. Clin J Am Soc Nephrol 2007; 2: 1186.

5. Iwashima Y, Horio T, Takami Y, et al. Effects of the creation of arteriovenous fistula for hemodialysis on cardiac function and natriuretic peptide levels in CRF. Am J Kidney Dis 2002; 40: 974.

6. Yigla M, Abassi Z, Reisner SA, Nakhoul F. Pulmonary hypertension in hemodialysis patients: an unrecognized threat. Semin Dial 2006; 19: 353.

7. Basile $\mathrm{C}$, Lomonte $\mathrm{C}$, Vernaglione $\mathrm{L}$, et al. The relationship between the flow of arteriovenous fistula and cardiac output in haemodialysis patients. Nephrol Dial Transplant 2008; 23: 282.

8. Movilli E, Viola BF, Brunori G, et al. Long-term effects of arteriovenous fistula closure on echocardiographic functional and structural findings in hemodialysis patients: a prospective study. Am J Kidney Dis 2010; 55: 682.

9. Ricciardi B., Badessa F., Saraniti A., et al. Biventricular stimulation and $\mathrm{HF}$ on line low flow in patient qith ischemic dilated cardiomiopathy NYHA-IV, Tenth Assisi European Meeting on CardioNefrology, vol. 8 pag 299 - Assisi Italy April 1-2-3 2004.

10. Ishani A, Collins AJ, Herzog CA, Foley RN. Septicemia, access and cardiovascular disease in dialysis patients: the USRDS Wave 2 study. Kidney Int 2005; 68: 311. 